\title{
Cognitive dysfunction in severe chronic obstructive pulmonary disease (COPD) with or without Long-Term Oxygen Therapy (LTOT)
}

\author{
Roberto W Dal Negro ${ }^{1,3^{*}}$, Luca Bonadiman ${ }^{1}$, Fernanda P Bricolo', Silvia Tognella ${ }^{2}$ and Paola Turco ${ }^{3}$
}

\begin{abstract}
Background: Chronic Obstructive Pulmonary Disease (COPD) is a progressive respiratory condition which can lead to comorbidities of variable severity, cognitive dysfunction included. The role of supplemental oxygen in preventing COPD-induced cognitive deterioration is still debated, but only episodically investigated. The aim of this study was to compare the cognitive pattern of hypoxemic COPD subjects treated with long-term oxygen (LTOT) to that of patients of comparable severity assuming oxygen on irregular basis, and to normal reference values.

Methods: Lung function, arterial blood gases, health status, and cognitive function measured by means of four psychometric tests focusing different domains of cognition (such as: MMSE, Clock test; TMT-A; TMT-B) were assessed in 146 well matched hypoxemic COPD patients (males $n=96,66 \%$; mean age $=70.5 \pm 12.9$ ). Seventy-three patients were assuming long-term oxygen (LTOT), while the remaining seventy-three were only using oxygen as needed (AN). Regarding statistics, $t$ test and ANOVA (Duncan test) were used to analyze data, assuming a $p<0.05$ as the lowest limit of significance.
\end{abstract}

Results: Even though all COPD patients showed a poorer psychometric profile vs corresponding normal reference values, LTOT patients showed a lower prevalence of severe deterioration in cognition. Also the extent of impairment was significantly lower in these patients when assessed by TMT-A and TMT-B ( $p<0.012$ and 0.001 , respectively), but not when measured by MMSE and Clock test (both $p=n s$ ). Several domains of cognition are variably affected by persistent hypoxemia in COPD patients. A panel of psychometric tools is needed for identifying the pattern of cognitive dysfunctions in these patients. Memory and attention (functions assessed by MMSE and Clock test) are only mildly-moderately affected, while visual processing, reproduction of numeric sequences, cognition flexibility, and shifting capacity (functions assessed by TMT-A and TMT-B) are much more deteriorated ( $p<0.012$ and $p<0.001$, respectively).

Conclusions: Only LTOT allows to preserve significantly $(p<0.022)$ cognitive functions from the COPD-induced deterioration. This assumption is of strategic value for COPD patients who are prescribed long-term oxygen because they frequently are not aware of the cognitive risks related to their condition.

Keywords: Cognition impairment, COPD, Long term oxygen treatment, Severe chronic obstructive pulmonary disease

\footnotetext{
* Correspondence: robertodalnegro@gmail.com

${ }^{1}$ National Centre for Respiratory Pharmacoeconomics and

Pharmacoepidemiology - CESFAR, Verona, Italy

${ }^{3}$ Research \& Clinical Governance, Verona, Italy

Full list of author information is available at the end of the article
} 


\section{Background}

Chronic Obstructive Pulmonary Disease (COPD) is a pathological condition of respiratory system prevailing since the $5^{\text {th }}$ decade of life and characterized by a high socioeconomic impact [1-3]. COPD can progressively affect the function of other organs (e.g. heart, vasculature, muscles, kidney, liver, gastro-enteric apparatus, and brain) leading to comorbidities of different severity $[4,5]$.

Impairment of cognitive functions is one of the effects of comorbidities related to COPD [6-9]. It has long been associated with severe pulmonary dysfunction [10-15], even if at a changing prevalence in different studies, because depending on the methods for assessing cognition and on the consistency of subjects' sample [16-20]. At present, some authors affirm that the lung function impairment assessed by means of $\mathrm{FEV}_{1}$ can be associated to a progressive cognitive function impairment in later life [15]. It has been demonstrated that hypoxemia per se can play a role in affecting cognition [21], and it might contribute to the onset and the progressive worsening of cognitive functions in subjects suffering from COPD, particularly in most severe cases [22]. Neverheless, other authors support the hypothesis that cognitive function is only mildly impaired without hypoxemia, and that cognitive dysfunction is higher in hypoxemic patients [9].

On the other hand, the role of supplemental oxygen in preventing the impairment of COPD-induced cognitive dysfunction is still debated.

Aim of the present study was to compare the cognitive dysfunction assessed in hypoxemic COPD subjects managed according to a strict protocol of home long-term oxygen (LTOT) to that of patients of comparable severity but assuming oxygen on irregular basis as needed (AN), and to normal reference values.

\section{Methods}

The participants enrolled in the present study were patients suffering from hypoxic or hypoxemic/hypercapnic COPD as defined in the GOLD guidelines [23]. Half patients were managed according to a regular program of home LTOT, while the remaining $50 \%$ of subjects used only oxygen as needed, without any predefined protocol, and in the vast majority of cases it was due to their adamant refusal of LTOT.

Each patient was assessed for: lung function (complete spirometry using CPFS/D - Medical Graphics Co.; Oak Grove Parkway, St. Paul, Minnesota, USA); arterial blood gases (using the ABL 735 Analyzer, Radiometer, Copenhagen, DK); health status (using the COPD Assessment test [CAT] questionnaire) [24,25]; and disability (using the Medical Research Council [MRC] dyspnoea scale) [26]. The prevalence of comorbidities was also assessed in all patients by means of Charlson morbidity index (C.M.I.) [27], together with their smoking history.
Cognition was evaluated in each patient by means of four validated psychometric questionnaires focusing different cognitive domains and characterized by different sensitivity. They were sequentially administered to all patients [22]: (1) the Mini Mental Status test (MMSE), which assesses spatial and time orientation, attention and calculation skills (normal score: $\geq 27$ points; moderate cognitive impairment: $24-18$ points; severe cognitive impairment: < 18 points [28], (2) the Clock Drawning test, which assesses memory, attention and symbolic representation (normal score: 7-10 points; limit for cognitive impairment: $\leq 6$ points) $[29,30]$; (3) the Trial Making test (TMT) $\mathrm{A}$, which assesses visual processing and reproduction of numeric sequences (limit for cognitive impairment: $\geq 94 \mathrm{sec}$ ) $[29,30]$; (4) the TMT B, which assesses cognition flexibility and shifting capacity (limit for cognitive impairment: $\geq 283 \mathrm{sec}$ ) [30].

Psychometric data obtained from normal age-matched controls were used as reference values [21].

Written informed consent was obtained from all subjects participating in the study (n. study approval 3898/2011).

\section{Statistics}

All data were expressed as means \pm SD (standard deviation). Comparisons between groups were carried out by means of $t$ test. Data of both groups were also compared to normal reference values by means of ANOVA (Duncan test). A p $<0.05$ was accepted as the lowest limit of statistical significance.

\section{Results}

The total sample consists of 146 subjects (males $\mathrm{n}=96$; $66 \%$; mean age $=70.5 \pm 12.9$ years ) suffering from hypoxemic (and/or hypercapnic) COPD. Seventy-three patients (males $n=49,67.1 \%$; mean age $=71.2 \pm 9.1$ ) were managed according to a strict protocol of regular home LTOT with liquid oxygen for 2.9 years $\pm 0,9$, with a mean oxygen use of $1.7 \mathrm{~L} / \mathrm{min} \pm 0.6$, for $>15$ hours/day, whereas the remaining 73 patients (males $n=48$; $65.7 \% \%$; mean age $=70.9 \pm 8.9$ years) used oxygen only as needed, on irregular basis.

The general characteristics of both groups are reported in Table 1. The two groups proved well matched regarding age, gender, smoking history, BMI, dyspnoea score, arterial blood gases, and lung function (all $t$ test comparisons $\mathrm{p}<0.05$ ). Also the mean C.M.I. was absolutely comparable in the two groups, being cardiovascular and metabolic comorbidities the most represented (such as $67.4 \%$ ) and equally prevailing in both groups.

The prevalence of severe cognitive impairment assessed in the two groups is reported in Figure 1. LTOT patients showed a significant lower prevalence of severe cognitive deterioration, independently of the psychometric 
Table 1 General characteristics of patients at baseline (means \pm SD) and their statistical comparison ( $t$ test)

\begin{tabular}{|c|c|c|c|}
\hline & without LTOT $(n=73$ ) & with LTOT $(n=73)$ & $p$ \\
\hline age (ys) & $70.9 \pm 8.9$ & $71.2 \pm 9.1$ & $\overline{\mathrm{ns}}$ \\
\hline FEV $_{\mathbf{1}} \%$ pred. & $40.2 \pm 10.3$ & $41.6 \pm 11.1$ & ns \\
\hline $\mathrm{FEV}_{1} / \mathrm{FVC}$ & $437 \pm 9.2$ & $44.2 \pm 10.2$ & ns \\
\hline BMI & $27.5 \pm 5.1$ & $27.9 \pm 6.4$ & ns \\
\hline Active smokers & $9.6 \% *$ & $6.8 \% *$ & ns \\
\hline Former smokers & $69.9 \% *$ & $72.6 \% *$ & ns \\
\hline $\mathrm{PaO}_{2}(\mathrm{mmHg})$ & $53.3 \pm 7.9$ & $54.2 \pm 8.3$ & ns \\
\hline $\mathrm{PaCO}_{2}(\mathrm{mmHg})$ & $45.8 \pm 9.9$ & $46.1 \pm 10.2$ & ns \\
\hline CAT score & $19.9 \pm 4.9$ & $18.8 \pm 5,6$ & ns \\
\hline MRC scale & $2.8 \pm 0.7$ & $2.7 \pm 0.8$ & ns \\
\hline C.M.I. & $4.2 \pm 2.0$ & $4.3 \pm 1.9$ & ns \\
\hline
\end{tabular}

*Smoking history is indicated in \% active or former smokers.

questionnaire used for measurements. In other words, all domains of cognition were significantly more deteriorated in AN patients.

Also the absolute extent of cognitive dysfunction was measured in both groups of patients, and corresponding data are reported in Table 2, together with the significance level of their statistical comparison. In particular, while the Clock test and the MMSE scores were equally informative in the two groups, the TMT-A and the TMT-B scores proved significantly different, and LTOT patients resulted significantly less impaired in their cognition compared to AN patients ( $\mathrm{p}<0.012$ and $\mathrm{p}<0.001$, respectively) (Table 2).

TMT-A and TMT-B proved the most discriminant psychometric tools and those worth of further analysis. Currently, as normal reference values for subjects aged 40-79 years are only available in the literature for the TMT-A and TMT-B [18], TMT-A and TMT-B scores obtained in the present study (range of age 65-75 years) were compared to corresponding normal reference values (Figures 2 and 3).

When compared to normals, the extent of cognitive dysfunction was significantly higher in all patients when assessed by TMT- A and TMT-B (ANOVA $\mathrm{p}<0.001$ for both comparisons). Nevertheless, LTOT patients proved significantly less limited in their cognition than AN patients $(t$ test $\mathrm{p}<0.022$ and $\mathrm{p}<0.05$, respectively).

\section{Discussion}

It is increasingly accepted that COPD is an inflammatory multicomponent, pathological condition which can affect several functions of different organs. Cognition represents one of these aspects, even if little attention has been paid to the effects of COPD on cognition in clinical practice, particularly in the presence of hypoxemia.

In general, the occurrence of significant limitations in cognitive functions was found to be associated with severe pulmonary dysfunction, particularly when chronically persisting, as in the case of COPD [12-15]. Recently, a specific study focused on these aspects and confirmed that both the prevalence and the extent of cognitive deterioration occurring in subjects with stable COPD of different severity are directly related to the severity of their pulmonary condition, hypoxemia included. In these cases, several domains of cognition are involved, and then several functions can be deteriorated, even substantially [22].

Hypoxemia has long been described as able to affect memory [21], even if this condition has been recently regarded as unlikely to account per se for the cognitive dysfunction in COPD [9]. On the other hand, its crucial role has been emphasized by other investigations which recently proved that the chronic persistence of hypoxemia is directly related to the extent of cognition impairment

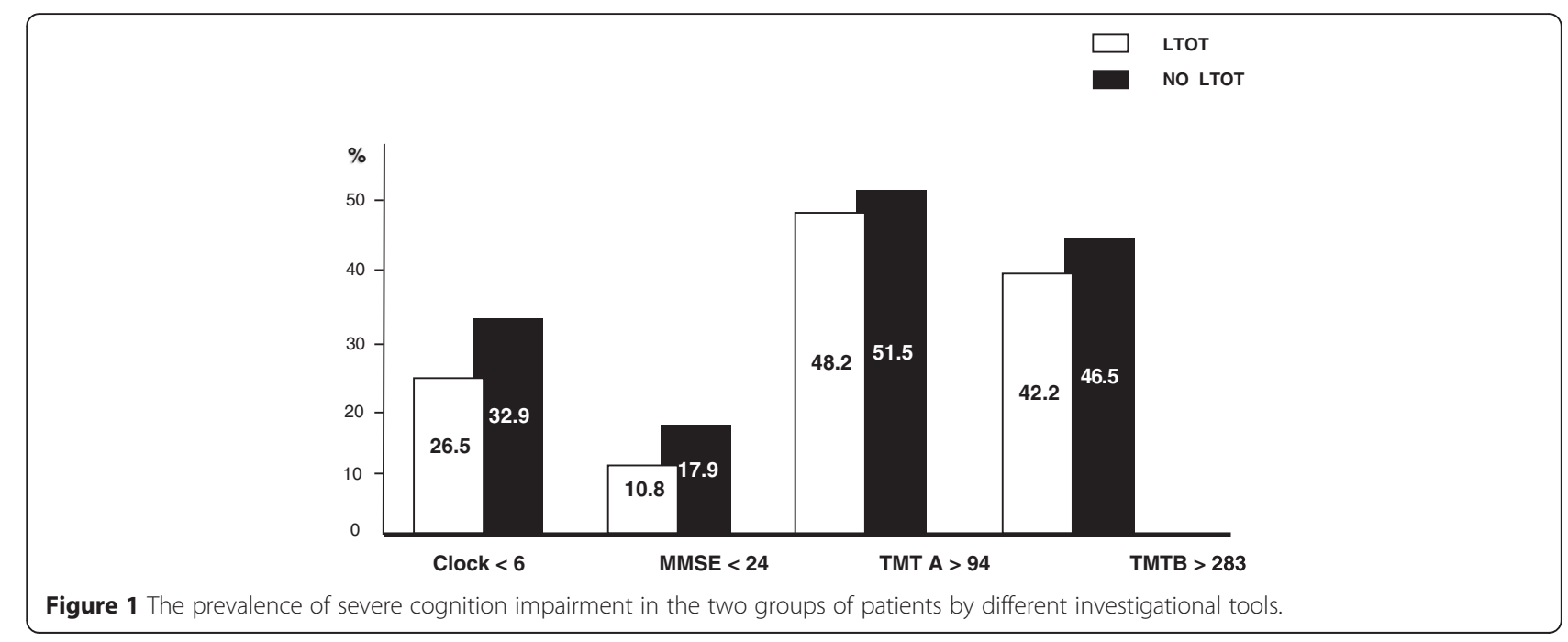


Table 2 Extent (mean \pm SD) of cognition impairment assessed in the two groups and significance of their comparison ( $t$ test)

\begin{tabular}{llll}
\hline test & LTOT $(\mathbf{n}=\mathbf{7 3})$ & NO LTOT $(\mathbf{n}=\mathbf{7 3})$ & $\mathbf{p}$ \\
\hline Clock test $<6$ & $4.2 \pm 1.7$ & $3.9 \pm 1.9$ & $\mathrm{~ns}$ \\
MMSE $<24$ & $20.1 \pm 3.1$ & $22.0 \pm 2.3$ & $\mathrm{~ns}$ \\
TMT-A $>94$ & $132.2 \pm 35.8$ & $155.3 \pm 52.5$ & 0.012 \\
TMT-B $>$ 283 & $322.1 \pm 36.2$ & $344.2 \pm 31.8$ & 0.001 \\
\hline
\end{tabular}

when investigated by means of the Montreat Cognitive Assessment [31]. Moreover, perception, attention, and short term memory has been described as significantly impaired in hypoxemic COPD patients in stable conditions, and the cause of this cognitive impairment has been related to neurophysiological events, such as the sustained presence of a decreased prefrontal cortex circulation [32].

Furthermore, also the intermittent, even if persistently occurring, hypoxemia which characterizes the obstructive sleep apnea syndrome (OSAS) has been suggested to have a role in developing cognitive impairment at variable degrees, particularly in ageing population (i.e. attention, episodic memory, working memory) [33].

When compared to corresponding normal reference values, a substantial deterioration in cognition was assessed in all COPD patients in present study, but according to a peculiar pattern. In particular, some cognitive functions resulted only mildly-moderately impaired (such as, those assessed by MMSE and Clock test), while others (such as, those assessed by TMT-A and TMT-B) resulted much more deteriorated. It would then be surmised that several domains of cognition are variably affected by persistent hypoxemia in COPD patients, such as: memory, attention, symbolic representation and visual processing, reproduction of numeric sequences, cognition flexibility, and shifting capacity. Long-term oxygen is presumed to consent a slower deterioration of all these cognitive

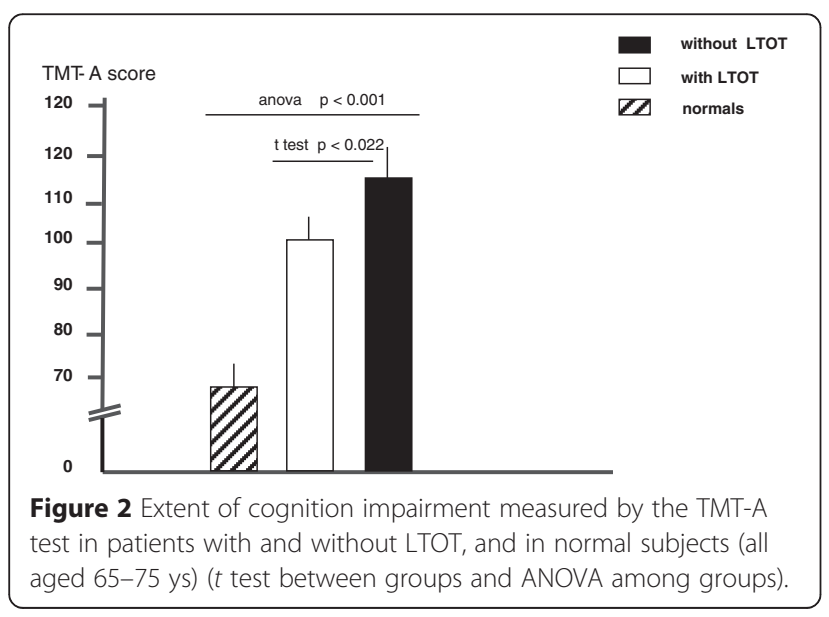

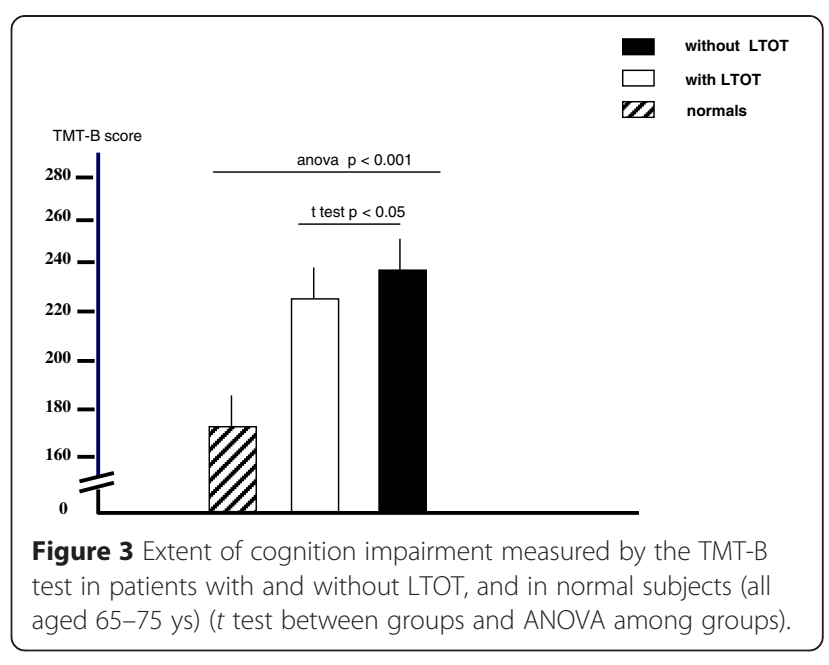

functions. Some of them (i.e. memory and attention) might be improved independently of the duration of oxygen assumption, while others (such as: visual processing, reproduction of numeric sequences, cognition flexibility, and shifting capacity), that likely are the most complex functions in neuro-psychological terms, are only sensitive to long-term oxygen treatment.

Consequently, a large panel of investigational instruments, covering a wide range of cognitive domains and differently sensitive is needed in order to specify the psycometric profile of these patients. Likely this is the reason why the occurrence of cognitive dysfunction was usually regarded as variable and depending on the psychometric test used $[34,35]$.

This particular aspect is also crucial when comparing the effect of different strategies of oxygen treatment (such as: regular OTLT vs oxygen as needed) on cognitive dysfunction. In the present study, the commonly used MMSE and Clock Drawing Test failed to discriminate the effects of home LTOT on cognition from those of oxygen as needed. Only TMT-A and TMT-B showed that long-term oxygen was much more effective in preserving these patients from cognitive dysfunctions, even if they still remain substantially limited compared to normal individuals. Consequently, different instruments provided with specific sensitivities should be used in order to highlight and differentiate the pattern of response to oxygen treatment.

The hypothesis that hypoxemia-induced cognitive deterioration might depend on complex neuro-physiological mechanisms in COPD (i.e. systemic inflammation and vascular damage affecting brain performance) seems to be confirmed by a study carried out according to a multiparametrical approach (such as by means of Short Test of Mental Status, transcranial Doppler ultrasonography, five cardiovascular tests, and a questionnaire of autonomic 


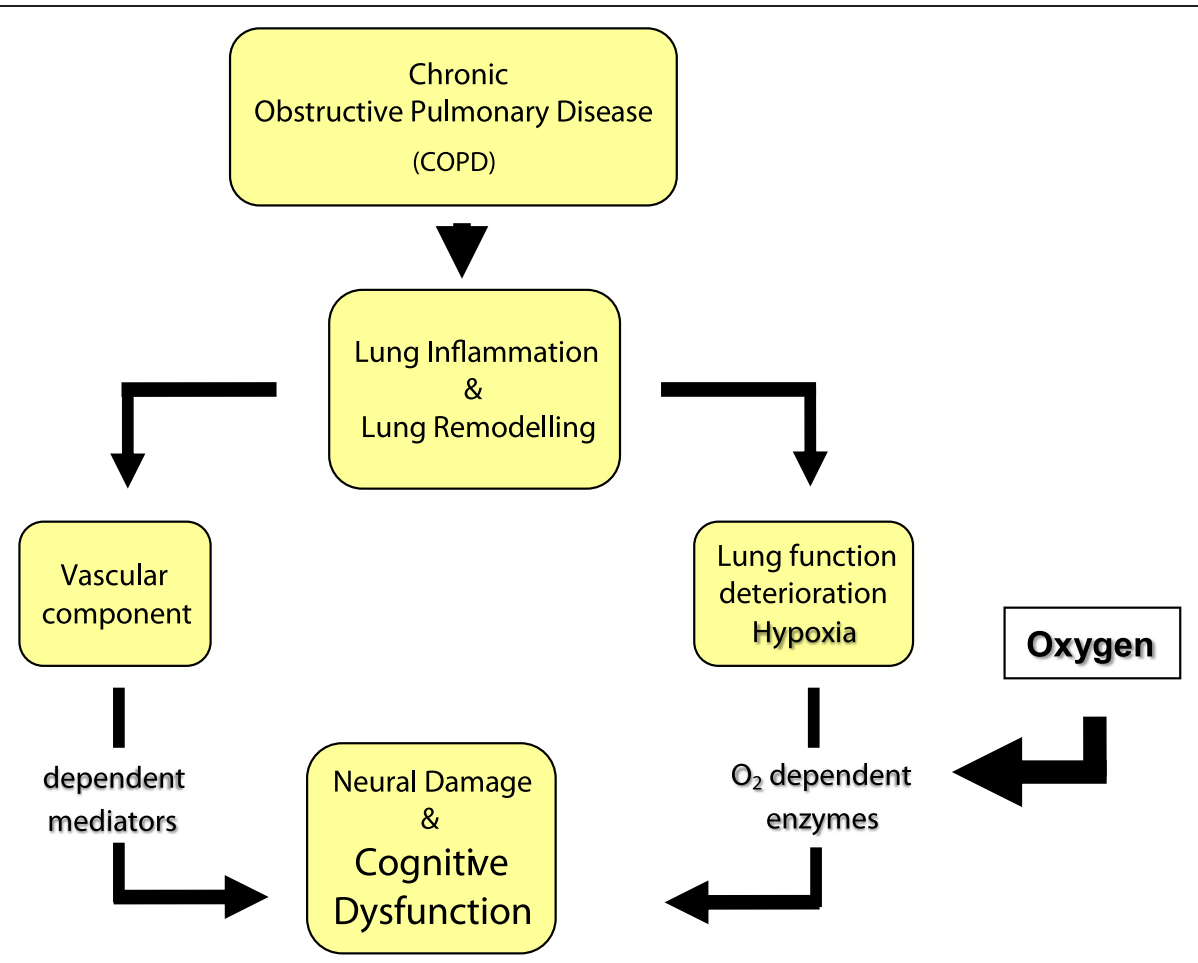

Figure 4 Deterioration of cognition in Chronic Obstructive Airway Disease: a comprehensive vision of the oxygen role in modulating the pathways of cognitive dysfunction.

function) in a small sample of hypoxemic COPD patients [36]. Also the effect of a three-month LTOT on the function of central and autonomic nervous system was investigated in this study. Patients' cognition was shown to improve proportionally to the amelioration of their neurological parameters, cerebral blood flow velocity, and autonomic function following LTOT, thus emphasizing the reversibility (at least partial) of the COPD-induced cognitive deterioration and the therapeutic effect of long-term oxygen from this point of view.

Persistent inflammation underlies chronic airflow limitation and is maximally effective in the severest conditions. As cognitive deterioration has been proved to be related to hypoxemia and to severity of airway obstruction [22], the active role of chronic inflammation in sustaining cognitive dysfunction can also be suggested in COPD. A comprehensive vision of the role of hypoxemia and of oxygen supplementation in modulating the pathways of cognitive dysfunction is reported in Figure 4.

In clinical terms, the strong evidence that cognition may be significantly deteriorated in hypoxemic COPD subjects contributes to explain why these subjects frequently demonstrate an insufficient self management and their adherence to therapeutic strategies frequently results inadequate. The psychometric profile of these subjects, as shown in the present study, can also contribute to explain their frequent requests for admission to health institutions and the consequent high economic impact.

\section{Conclusions}

In conclusion, focusing the effect of COPD on cognition is a relevant issue for all COPD patients, independently of their severity. The assumption that hypoxemic COPD subjects have a substantial cognitive dysfunction mainly affecting peculiar aspects of cognition is of strategic value for those COPD patients who are prescribed long-term oxygen because they frequently are not aware of the systemic risks related to their condition. They should be increasingly encouraged to accept LTOT, when needed, because this therapeutic strategy is effective in preserving their cognitive function from the deterioration induced by their chronic respiratory limitations, and in preventing progression of cognitive dysfunction.

\section{Competing interests}

The authors declare that they have no competing interests.

\section{Author details}

${ }^{1}$ National Centre for Respiratory Pharmacoeconomics and Pharmacoepidemiology - CESFAR, Verona, Italy. ${ }^{2}$ Lung Unit, Orlandi General Hospital, ULSS22 Regione, Veneto, Italy. ${ }^{3}$ Research \& Clinical Governance, Verona, Italy.

Received: 4 March 2015 Accepted: 30 March 2015

Published online: 19 April 2015 


\section{References}

1. Vestbo J, Hurd S, Agusti AG, Jones PW, Vogelmeier C, Anzueto A, et al. Global Strategy for the diagnosis, management and prevention of COPD: GOLD executive summary. Am J Respir Crit Care Med. 2013;187(4):347-65.

2. Sullivan SD, Ramsey SD, Lee TA. The economic burden of COPD. Chest. 2000:117(2 Suppl):5s-9.

3. Chapman KR, Mannino DM, Soriano JB, Vermeire PA, Buist AS, Thun MJ, et al. Epidemiology and costs of chronic obstructive pulmonary disease. Eur Respir J. 2006;27(1):188-207.

4. Ait-Khaled N, Enarson DA, Ottmani S, El Sony A, Eltigani M, Sepulveda R. Chronic airflow limitation in developing countries: burden and priorities. Int J Chron Obstruct Pulmon Dis. 2007;2(2):141-50

5. Eisner MD, Blanc PD, Yelin EH, Sidney S, Katz PP, Ackerson L, et al. COPD as a systemic disease: impact on physical functional limitations. Am J Med. 2008;121(9):789-96.

6. Calverly PM. Neuropsycological deficits in chronic obstructive pulmonary disease. Monaldi Arch Chest Dis. 1996;51(1):5-6.

7. Ambrosino N, Bruletti G, Scala V, Porta R, Vitacca M. Cognitive and perceived health status in patients with chronic obstructive pulmonary disease surviving acute or chronic respiratory failure: a controlled study. Intensive Care Med. 2002:28(2):170-7.

8. Antonelli-Incalzi C, Corsonello A, Troiano L, Pedone C, Acanfora D, Spada A, et al. Screening of cognitive impairment in chronic obstructive pulmonary disease. Dement Geriatr Cogn Disord. 2007;23(4):264-70.

9. Dodd JW, Getov SV, Jones W. Cognitive function in COPD. Eur Respir J. 2010;35(4):915-22.

10. Semenza C, Mondini S, Borgo F, Pasini M, Sgaramella MT. Proper names in patients with early Alzheimer's disease. Neurocase. 2003;9(1):63-9.

11. Ritchie K, Touchon J. Mild cognitive impairment: conceptual basis and current nosological status. Lancet. 2000;355(9199):225-8.

12. Grant I, Heaton RK, McSweeny AJ. Neurophysiological findings in hypoxemic chronic obstructive pulmonary disease. Arch Intern Med. 1982;142:1470-6.

13. Krzyzanowski M, Jedrichowski W, Wysocki M. Factors associated with the change in ventilator function and the development of chronic obstructive pulmonary disease in a 13-year follow-up of the Cracow study. Am Rev Respir Dis. 1986;134(5):1011-9.

14. Kuller LH, Ockene JK, Towsend M. The epidemiology of pulmonary function and COPD mortality in the Multiple Risk Factor Intervention Trial. Am Rev Respir Dis. 1989;140(3):S76-81.

15. Chyou PH, White LR, Yano K, Sharp DS, Burchfiel CM, Chen R, et al. Pulmonary function measures as predictors and correlates of cognitive functioning in later life. Am J Epidemiol. 1996;143(8):750-6.

16. Incalzi RA, Bellia V, Maggi S, Imperiale C, Capparella O, Catalano F, et al. Mild to moderate chronic airways disease does not carry an excess risk of cognitive dysfunction. Aging Clin Exp Res. 2002;14(5):395-401.

17. Petersen RC, Stevens JC, Ganguli M, Tangalos EG, Cummings JL, DeKosky ST. Practice Parameter: Early detection of dementia: Mild cognitive impairment (an evidence-based review). Report of the quality standards subcommittee of the American Accademy of Neurology. Neurology. 2001;56(9):1133-42.

18. Isoaho R, Puolijoki H, Huhti E, Laippala P, Kivela SL. Chronic obstructive pulmonary disease and cognitive impairment in the elderly. Int Psychogeriatr. 1996;8(1):113-25

19. Kozora E, Filley CM, Julian LJ, Cullum CM. Cognitive functioning in patients with chronic obstructive pulmonary disease and mild hypoxemia compared with patients with mild Alzheimer disease and normal controls. Neuropsychiatry Neuropsychol Behav Neurol. 1999;12(3):178-83.

20. Petersen RC, Smith GE, Waring SC, Ivnik RJ, Tangalos EG, Kokmen E. Mild cognitive impairment: clinical characterization and outcome. Arch Neurol. 1999;56(3):303-8.

21. Huppert FA. Memory impairment associated with chronic hypoxia. Thorax. 1982;37(11):858-60.

22. Dal Negro RW, Bonadiman L, Tognella S, Bricolo FP, Turco P. Extent and prevalence of cognitive dysfunction in chronic obstructive pulmonary disease, chronic non-obstructive bronchitis, and in asymptomatic smokers, compared to normal reference values. Chron Obstruct Pulmon Dis. 2014;9:675-83.

23. Global Initiative for Chronic Obstructive Lung Disease. Global strategy for the diagnosis, management, and prevention of chronic obstructive pulmonary disease. 2012. Available from: http://www.goldcopd.org/
Guidelines/guidelines-global-strategy-for-diagnosis-management-2012.html. Accessed December 10, 2014

24. Jones PW, Harding G, Berry P, Wiklund I, Chen WH, Kline Leikly N. Development and first validation of the COPD Assessment Test. Eur Respir J. 2009;34:648-54.

25. Dal Negro RW, Bonadiman L, Turco P. Sensitivity of the COPD Assessment Test (CAT questionnaire) investigated in a population of 681 consecutive patients referring to a lung clinic. The first Italian specific study. Multidiscip Resp Med. 2014;9:15-22.

26. Bestall JC, Paul EA, Garrod R, Garnham R, Jones PW, Wedzicha JA. Usefulness of the Medical Research Council (MRC) dyspnoea scale as a measure of disability in patients with chronic obstructive pulmonary disease. Thorax. 1999:54(7):581-6.

27. Charlson ME, Pompei P, Ales KL, MacKenzie CR. A new method of classifying prognostic comorbidity in longitudinal studies: development and validation. J Chronic Dis. 1987:40(5):373-83.

28. Folstein MF, Folstein SE, McHugh PR. "Mini-mental state". A practical method for grading the cognitive state of patients for the clinician. $J$ Psychiatr Res. 1975;12(3):189-98.

29. Brown EC, Casey A, Fisch RI, Neuringer C. Trial making test as a screening device for the detection of brain damage. J Consult Psychol. 1958:22:469-74.

30. Giovagnoli AR, Del Pesce M, Mascheroni S, Simoncelli M, Laiacona M, Capitani E. Trail making test: normative values from 287 normal adult controls. Ital J Neurol Sci. 1996;17(4):305-9.

31. Qian H, Lin H, Li Y. Assessment of cognition and associated factors in patients with stable chronic obstructive pulmonary disease. Zohngua Jie He He Hu Xi Za Zhi. 2014;37:769-73.

32. Fujimoto S, Kobayashi S, Yoshikawa T, Hirata K. Cognitive function in patients with chronic obstructive pulmonary disease. Nihon Rinsho. 2014;72:721-5

33. Gagnon K, Baril AA, Gagnon JF, Fortin M, Decary A, Lafond C, et al. Cognitive impairment in obstructive sleep apnea. Pathol Biol. 2014;62:233-40.

34. Pleis JR, Leithbridge-Cejku M. Summary Health Statistics for U.S. adults. National Health Interview Survey. 2006. Vital Health Stat. 2007;10:20-3.

35. Anstey KJ, Windsor TD, Jorm AF. Association of pulmonary function with cognitive performance in early, middle and late adulthood. Gerontology. 2004;50(4):230-4

36. Hjalmarsen A, Waterloo K, Dahl A, Jorde R, Viitanen M. Effect of long-term oxygen therapy on cognitive and neurological dysfunction in chronic obstructive pulmonary disease. Eur Neurol. 1999;42:27-35.

\section{Submit your next manuscript to BioMed Central and take full advantage of:}

- Convenient online submission

- Thorough peer review

- No space constraints or color figure charges

- Immediate publication on acceptance

- Inclusion in PubMed, CAS, Scopus and Google Scholar

- Research which is freely available for redistribution

Submit your manuscript at www.biomedcentral.com/submit
C Biomed Central 